\title{
Kızıl Tilkilerde (Vulpes vulpes) Arteria Celiaca ve Dalları Üzerinde Bir
}

\author{
Çalışma* \\ Gülseren Kırbaş Doğan ${ }^{1, a, * *}$, Semine Dalga ${ }^{1, b}$, Yalçın Akbulut ${ }^{2, c}$, Kadir Aslan $^{1, d}$ \\ ${ }^{1}$ Kafkas Üniversitesi, Veteriner Fakültesi, Anatomi Anabilim Dalı, Kars, Türkiye \\ ${ }^{2}$ Kafkas Üniversitesi, Tıp Fakültesi, Anatomi Anabilim Dalı, Kars, Türkiye \\ aORCID: 0000-0003-3770-9956, '⿳O口ORCID: 0000-0001-7227-2513, ' ORCID: 0000-0003-4661-2224, dORCID: 0000-0002-7617-0175 \\ Geliş Tarihi: 15.05.2019 \\ Kabul Tarihi: 13.11.2019

\begin{abstract}
Özet: Sunulan çalışmada kızı tilkilerde arteria celiaca ve dallarının araştııılması amaçlandı. Çalışmada 6 adet kızı tilki kullanıldı. Arteria celiaca'nın 1. lumbal vertebra hizasında aorta abdominalis'ten ayrıldığı ve ilk ayrılan dalın ise arteria hepatica olduğu belirlendi. Arteria hepatica'dan ortalama $8.48 \mathrm{~mm}$ sonra kalın olan arteria lienalis ve daha ince olan arteria gastrica sinistra'nın ortak bir kök halinde başlangıç aldığı görüldü. Arteria gastroduodenalis'in arteria pancreaticoduodenalis cranialis ve arteria gastroepiploica dextra’ya ayrılarak sonlandığı görüldü. Arteria lienalis'ten biri dalağın extremitas dorsalis'ine, diğeri extremitas ventralis'ine giden iki damar ayrıldığı tespit edildi. Extremitas dorsalis'e giden arteria lienalis'in arteriae gastricae breves dallarının midenin fundus bölümünde sonlandığı görüldü. Arteria lienalis'in extremitas ventralis'e giden arteria gastroepiploica sinistra dalı önce 3 dala daha sonra çok sayıda dallara ayrılarak midenin curvatura major'unda arteria gastrica dextra ile anastomoz yaptığı belirlendi. Extremitas ventralis'e giden ana daldan pankreas'ı besleyen 2-3 ince dalın ayrıldığı görüldü. Sonuç olarak sunulan bu araştırmanın bulgularının evcil ve yaban hayvanı olan carnivorlar üzerinde yapılacak olan splenektomi, mide ve karaciğer operasyonlarına destek olacağı düşünülmektedir.

Anahtar Kelimeler: Anatomi, Arteria celiaca, Carnivor, Kızıl tilki.
\end{abstract}

\section{A Study on Arteria Celiaca and Branches in Red Foxes (Vulpes vulpes)}

Abstract: This study aimed to conduct a research on arteria celiaca and its branches in Red foxes (Vulpes vulpes). Six red foxes were used. It was determined that arteria celiaca was separated from the aorta abdominalis in the line of first lumbal vertebrae and arteria hepatica was the first branch derivated from arteria celiaca. The thick branch (arteria lienalis) and the thin branch (arteria gastrica sinistra) were found to derivate as a common root with a mean distance of $8.48 \mathrm{~mm}$ from arteria hepatica. Arteria gastroduodenalis was found to be terminated by giving branches such as arteria pancreaticoduodenalis cranialis and arteria gastroepiploica dextra. Arteria lienalis was found to have separated two vessels leading to the extremitas dorsalis and the extremitas ventralis of spleen. The branches of the arteria lienalis called as arteriae gastricae breves were found to terminated in the fundus part of the stomach are by leading to the extremitas dorsalis. Arteria gastroepiploica sinistra, which is the branch of arteria lienalis leading to the extremitas ventralis, was found to gave first 3 branches and then divided into a large number of branches by giving anastomosis with arteria gastrica dextra in the line of curvature of the stomach. It was seen that 2-3 thin branches feeding the pancreas were derivated from the main branch of the extremitas ventralis. We believe that the results of this study will provide further data supporting splenectomy, stomach and liver operations on domestic and wild carnivores.

Keywords: Anatomy, Arteria celiaca, Carnivora, Red fox.

\section{Giriş}

Veteriner anatomi alanında, karşılaştırmalı hayvan anatomisi değişik hayvan ırkları ve türleri arasındaki farklılıkları ortaya koyma açısından öneme sahiptir. Evcil hayvanlarda tür ve ırk bazında bu farklılıklar çoğunlukla ortaya konulmuştur (Gezici ve Dursun, 1999; Silva ve ark., 2018; Yousefi, 2017). Bir yaban hayvanı olan Kızıl tilki köpekgiller (Canidae) familyasındadır. Kızıl tilki'nin Avrupa, Asya, Kuzey Afrika ve Amerika kıtasında yaşayan türleri vardır. Tilkiler kulakları sivri ve büyük, kuyruğu uzun, zekâ ve hilesiyle ünlü bir etçildir (Şekil 1) (Larivière ve Pasitschniak-Arts, 1996).

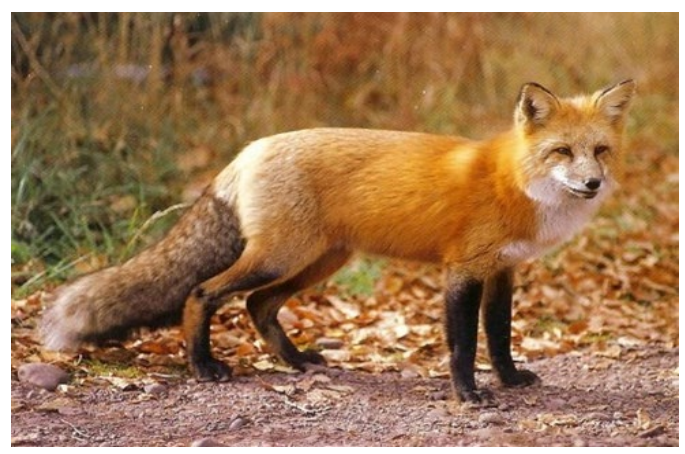

Şekil 1. Kızıl tilki (Anonim, 2018). 
Canlı için gerekli olan besin maddelerinin hücrelere taşınması ve oluşan metabolizma artıklarının uzaklaştırılması dolaşım sistemi sayesinde olmaktadır. Kalp ve damarlar dolaşım sisteminin en önemli elemanlarıdır. Bu önem dahilinde farklı hayvan türlerinde organları besleyen damarların seyri hayati önem taşımaktadır (König ve Liebich, 2015).

Arteria (a.) celiaca, diaphragma'nın crus'ları arasında, son sırt veya ilk bel omuru düzeyinde aorta abdominalis'in alt yüzünden tek olarak (Sission ve Grossman, 1964) çıkan ilk visceral daldır (Dursun, 2008; Miller, 1964). Mide, karaciğer, dalak ve pankreas ile ince barsakların başlangıç kesimlerinin beslenmesini sağlayan ortak bir köktür. Yaklaşık $4 \mathrm{~mm}$ çapında ve $20 \mathrm{~mm}$ uzunluğundadır (Nickel ve ark., 1981). Arteria gastrica sinistra, a. hepatica ve a. lienalis olmak üzere üç dala ayrılır (Dursun, 2008; König ve Liebich, 2015; Miller, 1964; Sission ve Grossman, 1964). Arteria lienalis a. celiaca'dan ayrılan üç dalın en kalın olanıdır. Bazı carnivorlarda üçüncü dal olarak değil a. celiaca'nın devamı olarak tanımlanır. Arteria gastrica sinistra ise bu dalların en incesidir (Dursun, 2008; Sission ve Grossman, 1964).

Diğer carnivor türlerinde a. celiaca üzerinde yapılan çalışmalar (Borelli ve Boccalleti, 1974; Yılmaz ve ark., 2004; Yousefi, 2017) mevcut olmasına rağmen yaban hayvanı olan kızıl tilkilerde böyle bir çalışmaya rastlanmadı. Bu nedenle gerek ilgili bölge üzerinde yapılacak uygulamalarda, gerekse kızıl tilki ile diğer evcil ve yabani carnivorlar arasında yapılacak karşılaştırma işlemlerinde ihtiyaç duyulacak bilgi eksikliğini gidermek için bu çalışma planlandı.

\section{Materyal ve Metot}

Tarım ve Orman Bakanlığı, Doğa Koruma ve Milli Parklar Genel Müdürlüğü'nden gerekli izinler alındıktan sonra bu çalışma yürütüldü (E.892955). Çalışmada Kafkas Üniversitesi Yaban Hayatı Kurtarma ve Rehabilitasyon Merkezi'nden temin edilen, cinsiyet farkı gözetilmeksizin erişkin 6 adet kızıl tilki kullanıldı. Çalışma materyalini, ateşli silahlarla veya trafik kazalarıyla ciddi şekilde yaralanmış ve tüm müdahalelere rağmen kurtarılamamış tilkiler oluşturdu. Aorta abdominalis tespit edildikten sonra ligatüre edildi. Tilkilerin arteriyel sistemine aorta'dan önce fizyolojik tuzlu su sonra kırmızı boya ile renklendirilmiş latex (Polytek marka) perfüze edildi. 10 gün soğutucuda bekletildi. Latex'in katılaşması sağlandıktan sonra arterler diseke edildi. Diseksiyonla elde edilen bulgular Kodak Digital Camera M 320 fotoğraf makinası ile fotoğraflandı. Damarların isimlendirilmesinde Nomina Anatomica Veterinaria (2017) referans alındı. Ölçümler damarların ayrılma noktasından digital kumpas (Stainless 0-150 mm) kullanılarak alındı. Damarların uzunluk ve çaplarının ortalama ve standart sapma değerleri SPSS (20.0 version) paket programında hesaplandı.

\section{Bulgular}

Arteria celiaca'nın birinci lumbal omur hizasında aorta abdominalis'ten ayrıldığı, a. celiaca'dan ilk ayrılan dalın ise a. hepatica olduğu belirlendi. Arteria celiaca'nın dalı olan a. hepatica'dan ortalama $8.48 \mathrm{~mm}$ sonra kalın olan a. lienalis ve daha ince olan a. gastrica sinistra'nın ortak bir kök halinde başlangıç aldığı görüldü (Şekil 2).

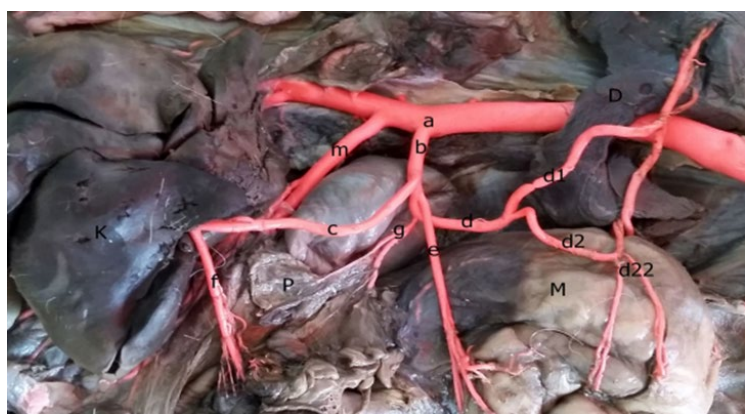

Şekil-2. Kızıl tilkilerde arteria celiaca'nın dallanması (K: Hepar, D: Lien, M: Gaster, P: Pancreas, a: Aorta abdominalis, b: a. celiaca, c: a. hepatica, d: a. lienalis, e: a. gastrica sinistra, f: a. gastroduodenalis, g: rami pancreatici, d1: a. lienalis'in dalağın extremitas ventralis'ine giden dalı, d2: a. lienalis'in dalağın extremitas dorsalis'ine giden dalı, d22: aa. gastricae breves, $\mathbf{m}$ : a. mesenterica cranialis)

Arteria hepatica'nın karaciğer ve mide sınırında iki dala ayrıldığı, bu dallardan birinin ramus dexter lateralis, ramus dexter medialis ve ramus sinister olmak üzere üç dala ayrılarak karaciğeri beslediği tespit edildi. Diğer dalların ise mide ile onikiparmak bağırsağı arasından geçip midenin curvatura major'unda dağılan a. gastrica dextra ve a. gastroduodenalis olduğu belirlendi. A. gastroduodenalis'in a. pancreaticoduodenalis cranialis ve a. gastroepiploica dextra'ya ayrılarak sonlandığı görüldü. Arteria lienalis'ten biri dalağın extremitas dorsalis'ine, diğeri extremitas ventralis'ine giden iki damar ayrıldığı tespit edildi. Extremitas dorsalis'e giden a. lienalis'in aa. gastricae breves isimli dalları midenin fundus bölümünde sonlandığı görüldü. Arteria lienalis'in extremitas ventralis'e giden a. gastroepiploica sinistra dalı önce üç dala daha sonra 7 dala ayrılarak midenin curvatura major'unda a. gastrica dextra ile anastomoz yaptığı belirlendi. Extremitas ventralis'e giden ana daldan pankreas'ı besleyen birkaç ince dalın ayrıldığı görüldü. Arteria gastrica sinistra'dan ilk olarak başlangıç alan rami esophagei'nin, 
esophagus'un pars abdominalis bölümünü beslediği tespit edildi.

Arteria celiaca'nın ortalama uzunluğu $14.37 \pm 2.55 \mathrm{~mm}$, çapı ise $2.11 \pm 0.23 \mathrm{~mm}$ olarak ölçüldü (Tablo 1).

Tablo 1. Kızıl tilkilerde a. celiaca'ların uzunluk ve çapları.

\begin{tabular}{rrr}
\hline A. celiaca & Uzunluk(mm) & \multicolumn{1}{c}{ Çap(mm) } \\
\hline KT1 & 11.50 & 2.45 \\
KT2 & 18.85 & 1.95 \\
KT3 & 12.50 & 1.90 \\
KT4 & 15.12 & 2.30 \\
KT5 & 14.05 & 1.92 \\
KT6 & 14.19 & 2.15 \\
\hline
\end{tabular}

KT: KızIl tilki

Arteria hepatica, a. lienalis ve a. gastrica sinistra'nın ortalama çap değerleri sırasıyla $1.64 \pm 0.13 \mathrm{~mm}, 1.33 \pm 0.91 \mathrm{~mm}$ ve $1.10 \pm 0.08 \mathrm{~mm}$ olarak ölçüldü (Tablo 2).

Tablo 2. Kızıl tilkilerde a. celiaca'nın dallarının çapları.

\begin{tabular}{cccc}
\hline $\begin{array}{c}\text { Kızıl } \\
\text { tilkiler }\end{array}$ & $\begin{array}{c}\text { A.hepatica'nın } \\
\text { çapı (mm) }\end{array}$ & $\begin{array}{c}\text { A.lienalis'in } \\
\text { çapı (mm) }\end{array}$ & $\begin{array}{c}\text { A.gastrica } \\
\text { sinistra'nın } \\
\text { çapı (mm) }\end{array}$ \\
\hline KT1 & 1.60 & 1.45 & 1.10 \\
KT2 & 1.88 & 1.20 & 0.95 \\
KT3 & 1.53 & 1.30 & 1.15 \\
KT4 & 1.64 & 1.28 & 1.17 \\
KT5 & 1.55 & 1.34 & 1.13 \\
KT6 & 1.62 & 1.41 & 1.10 \\
\hline
\end{tabular}

KT: Kızıl tilki

\section{Tartışma ve Sonuç}

Arteria celiaca, diaphragma'nın crus'ları arasında, on üçüncü thoracal veya ilk lumbal vertebra (Abidu-figueiredo ve ark., 2008; Nickel ve ark., 1981) düzeyinde aorta abdominalis'in alt yüzünden tek olarak (Abidu-figueiredo ve ark., 2005; Awal ve ark. 2001; Sission ve Grossman, 1964) çıkan ilk visceral daldır (Dursun, 2008; Miller, 1964). Kızıl tilkilerde de a. celiaca'nın birinci lumbal vertebra hizasında aorta abdominalis'ten ayrılan ilk dal olduğu görüldü. Brezilya dövüş tilkisinde de a. celiaca ilk daldır (Silva ve ark., 2018). Fakat porsukta ilk visceral dal a. phrenica caudalis'tir (Yılmaz ve ark., 2004). Arteria celiaca mide, karaciğer, dalak ve pancreas ile ince barsakların başlangıç kesimlerinin beslenmesini sağlayan ortak köktür. Arteria gastrica sinistra, a. hepatica ve a. lienalis olmak üzere üç dala ayrılır (Dursun, 2008; Miller, 1964; Sission ve Grossman, 1964). A. lienalis, a. celiaca'dan ayrılan üç dalın en kalını, a. gastrica sinistra en incesi (Dursun, 2008; Sission ve Grossman, 1964) olarak bildirilirken kızıl tilkilerde a. hepatica en kalını (1.64 $\mathrm{mm})$, a. gastrica sinistra en incesi $(1.10 \mathrm{~mm}$ ) olarak dikkat çekti. A. celiaca'nın en kalın dalı bulgumuz uyuşmaz iken en ince dal bulgumuz aynıdır. Arteria gastrica sinistra; kedide (Nickel ve ark., 1981) ve Yeni Zelanda tavşanında (Abidu-figueiredo ve ark., 2008) a. celiaca'dan, köpekte ise a. lienalis'ten köken alır (Nickel ve ark., 1981). Kedilerde yapılan başka bir araştırmada (Borelli ve Boccalleti, 1974) a. celiaca'dan ayrılan ilk dalın çoğunlukla (\%90) a. hepatica olduğu görülmüştür. Bazı durumlarda (\%8) a. hepatica ve a.gastrica sinistra'nın ortak bir kök olarak çıktığı da belirlenmiştir. Sadece bir hayvanda a. hepatica, a. lienalis ve a. gastrica sinistra'nın ortak bir kökten çıktığı tespit edilmiştir. Köpeklerde yapılan çalışmada (Abidu-figueiredo ve ark., 2005) \%56.7 oranında a. hepatica'nın tek dal olarak truncus gastrolienalis'in de ayrı bir dal olarak çıktığı belirlenirken \%33.3'ünde üç damarın da tek bir kökten ayrıldığı rapor edilmiştir. Köpeklerdeki yüksek orana (Abidu-figueiredo ve ark., 2005; Awal ve ark. 2001) benzer olarak merkepte de aynı dallanma tespit edilmiştir (Karakurum ve Dursun, 2010). Kangal köpeklerinde ise üç damarın da a. celiaca'dan ayrı ayrı çıktığı belirlenmiştir (Gezici ve Dursun, 1999). Bizim çalışmamızda tüm materyallerde a. celiaca'dan ilk olarak a. hepatica'nın çıktığı, a. lienalis ve a. gastrica sinistra'nın ise tek kökten çıktığı görüldü. Bulgumuzun literatürde (Abidu-figueiredo ve ark., 2005; Borelli ve Boccalleti, 1974) bildirilen damarların çoğunun seyriyle benzer olduğu anlaşılmaktadır.

Köpekte a. lienalis'in; ramus pancreaticus, a. gastricae breves ve a. gastroepiploica sinistra dallarına ayrıldığı rapor edilmiştir (Budras ve ark., 2009; Dursun, 2008; Evans ve de Lahunta, 2013; N.A.V., 2017). Kızıl tilkilerde a. lienalis'ten iki damar ayrıldığı tespit edildi. Bunlardan extremitas dorsalis'e giden a. lienalis'in aa. gastricae breves isimli dalları, a. lienalis'in extremitas ventralis'e giden a. gastroepiploica sinistra dalı önce 3 dala daha sonra çok sayıda dallara ayrılarak midenin curvatura major'unda a. gastrica dextra ile anastomoz yaptığı gözlendi. Extremitas ventralis'e giden ana daldan pankreas'ı besleyen 2-3 ince dalın (rami pancreatici) ayrıldığı tespit edildi. Ağaç sansarında a. lienalis'in üç dala ayrıldığı bildirilirken (Yousefi, 2017) kızıl tilkilerde iki dala ayrıldığı görüldü.

Literatürde carnivor'larda a. hepatica'nın; ramus dexter lateralis, ramus dexter medialis, ramus sinister, a. gastrica dextra ve a. gastroduodenalis'e ayrıldığı, a. gastroduodenalis'in ise a. pancreaticoduodenalis ve a. gastroepiploica 
dextra'yı verdiği bildirilmiştir. (Budras ve ark., 2009; Dursun, 2008; N.A.V., 2017). Kızıl tilkilerde de a. hepatica'nın ramus dexter lateralis, ramus dexter medialis, ramus sinister, a. gastrica dextra ve a. gastroduodenalis'e ayrıldığı fakat a. pancreaticoduodenalis cranialis ve a. gastroepiploica dextra'nın a. gastrica dextra'nın devamı şeklinde olduğu ortaya konuldu.

A. celiaca'nın ortalama uzunluğu carnivorlarda yapılan bazı çalışmalarda 20 mm (Dyce, 1987; Evans ve Lahunta, 2013; Nickel ve ark., 1979), sincapta (Akgöl ve Aydın, 2016) $30 \mathrm{~mm}$, köpekte yapılan çalışmalarda $9.8 \mathrm{~mm}$ (Abidu-figueiredo ve ark., 2005) ile $16 \mathrm{~mm}$ (Ghiringhelli ve ark. 2016), Alman çoban köpeğinde 13-15 mm (Özcan ve ark., 2001), Kangal köpeğinde 20-25 mm (Gezici ve Dursun, 1999) olarak belirlenmiştir. Kızıl tilkilerde bu değer $14.37 \pm 2.55 \mathrm{~mm}$ olarak ölçüldü. Literatür taramalarında a. celiaca'nın ortalama uzunluğu minimum 9.8 mm (Abidu-figueiredo ve ark., 2005) maksimum $30 \mathrm{~mm}$ (Akgöl ve Aydın, 2016) olarak ölçülmüştür. Minimum ve maksimum değerler dikkate alındığında kızıl tilkilerde a. celiaca uzunluğunun ortalama değerler arasında olduğu kanısına varıldı. Arteria celiaca'nın ortalama çapı köpeklerde $5.33 \mathrm{~mm}$ (Ghiringhelli ve ark. 2016) iken kızıl tilkilerde $2.11 \mathrm{~mm}$ olarak belirlendi. Roza ve ark., (2012)'nın köpekte yaptığı çalışmada damar uzunlukları a. hepatica'nın $27 \mathrm{~mm}$, a. lienalis'in 21 $\mathrm{mm}$, a. gastrica sinistra'nın $20 \mathrm{~mm}$ olarak tespit edilmiştir. Kızıl tilkilerde damar çapları uzunluğu sırasıyla $1.64 \pm 0.13,1.33 \pm 0.91$ ve $1.10 \pm 0.08 \mathrm{~mm}$ olarak belirlendi. Damar çaplarının uzunluk değerleri farklı olmasına rağmen en kalın damarların sırasıyla a. hepatica, a. lienalis ve a. gastrica sinistra olması bulgumuz aynıydı.

Carnivor'lar hiperaktif hayvanlar oldukları için travma ve trafik kazaları çok sık görülmektedir (Björnstig ve ark. 1991). Bu vakalar sonucunda dalak parçalanabilmekte ve splenektomi operasyonu gerekmektedir (Berland ve VanDyke). Splenektomi sırasında mideye gelen kan miktarının azalmasını engellemek için a. lienalis'in seyrini bilmek gerekmektedir. Evcil hayvanlara müdahale sırasında a. lienalis'in seyri bilindiği için sorun yaşanmamaktadır (Awal ve ark. 2001, Borelli ve Boccalleti 1974, Ghiringhelli ve ark. 2016). Fakat yaban hayvanlarından kızıl tilkilerde bu seyir bilinmemektedir. Sunulan çalışmada kızıl tilkilerde a. celiaca'nın morfometrisi, dalları ve seyirleri belirlendi. Sonuç olarak sunulan bu araştırmanın bulgularının evcil ve yaban hayvanı olan carnivor'lar üzerinde yapılacak olan splenektomi, mide ve karaciğer operasyonlarına destek olacağ düşünülmektedir.

\section{Kaynaklar}

Abidu-figueiredo M, Dias GP, Cerutti S, Carvalho-de-souza B, Maia RS, Babinski MA, 2005: Variations of celiac artery in dogs: Anatomic study for experimental, surgical and radiological practice. Int J Morphol, 23, 37-42.

Abidu-figueiredo M, Xavier-Silva B, Cardinot TM, Babinski MA, Chages NA, 2008: Celiac artery in New Zealand rabbit: Anatomical study of its origin and arrangement for experimental research and surgical practice. Pesq Vet Bras, 28, 237-240.

Akgöl B, Aydın A, 2016: Sincaplarda (Sciurus vulgaris) arteria celiaca'nın dağııımı. Journal of Bahri Dagdas Animal Research, 5, 1-11.

Anonim, 2018: http://altinotu.blogspot.com/2012/12/kzltilki-red-fox.html, Erişim tarihi; 09.05.2018.

Awal MA, Asaduzzaman M, Anam MK, Prodhan MAA, Kurohmaru M, 2001: Arterial supply to the stomach of Indigenous dog (Canis familiaris) in Bangladesh. Exp Anim, 50, 349-352.

Berland LL, VanDyke JA, 1985: Decreased splenic enhancement on $\mathrm{CT}$ in traumatized hypotensive patients. Radiology, 156 (2), 469-47.

Budras KD, Fricke W, Richter R, 2009: Veteriner Anatomi Atlası (Köpek). Çev.: Kürtül í, Düzler A, Çevik Demirkan A, Atalgın ŞH, Bozkurt EÜ, Aksoy G, Eyol E, Özcan S, Sancak AA, Medipress matbaacılık yayıncılık, Ltd. şti., Malatya, 62.

Borelli V, Boccalleti D, 1974: Ramificação das aa. celíaca e mesentérıca cranial no gato (Felis catus domestica). Rev Fac Med Vet Zootec Univ, 11, 263-270.

Björnstig U, Eriksson A, Ornehult L, 1991: Injuries caused by animals. Injury, 22, 295-298

Dursun N, 2008: Veteriner Anatomi II, Medisan Yayınevi, Ankara, 242-245.

Dyce KM, 1987: Textbook of Veterinary Anatomy. W.B. Saunders Company, New York.

Evans HE, de Lahunta A, 2013: Miller's Anatomy of the Dog. Fourth edition WB Sunders Company, Philadelphia, 367-386.

Gezici M, Dursun N, 1999: Kangal köpeğinde a. celiaca'nın dağılımı. Vet Bil Derg, 15, 15-21.

Ghiringhelli M, Brizzola S, Acocella F, Coretti D, 2016: Clinical anatomy of the celiac trunk in the dog: application for elective surgery or surgical emergency. S.I.S.V.E.T.-S.I.C.V., Palermo.

Karakurum E, Dursun N, 2010: Merkepte (Equus asinus L.) midenin arterial vaskularizasyonu. Kafkas Univ Vet Fak Derg, 16, 143-418. DOI:10.9775/kvfd.2009.845.

König HE, Liebich HG, 2015: Veteriner Anatomi (Evcil Memeli Hayvanlar), Systema cardiovasculare, Çev.: Özgel Ö, Halıgür A, Karakurum E, Medipres, 6. baskı, 470.

Larivière $S$, Pasitschniak-Arts $M$, 1996: Mammalian Species Vulpes vulpes. A S M. 537, 1-11.

International Committee on Veterinary Gross Anatomical Nomenclature. Nomina Anatomica Veterinaria (NAV), 2017: World Association of Veterinary Anatomists, 6th ed, Hanover, Germany.

Miller ME, 1964: Anatomy of the Dog. Philedelphia : W. B. Saunders Company, 345-349. 
Nickel R, Schummer A, Seiferle E, 1981: The Anatomy of the Domestic Animals. Vol. 3, Verlag Paul Parey, Berlin, 159.

Özcan S, Kürtül i, Aslan K, 2001: Alman çoban köpeklerinde midenin arteriyel vaskülarizasyonu. istanbul Üniv Vet Fak Derg, 27(2), 487-494.

Roza MS, Marinho GC, Pereira JA, Salvador-Gomes M, Abidu-Figueiredo M, 2012: Celiac artery with a pulmonary branch in dog: a rare variation. $J$ Morphol Sci, 29(4), 253-255.

Silva DRS, Silva MD, Assunção MPB, Chacur EP, Silva DCO, Barros RAC, Silva Z, 2018: Anatomy of the abdominal aorta in the hoary fox (Lycalopex vetulus, Lund, 1842). Braz J Vet Res Anim Sci, 55(4), 1-6.

Sission SB, Grossman JD,1964: The Anatomy of the Domestic Animals. 4th edition, Philadelphia London, W. B. Saunders Company, 673-675.
Yılmaz S, Atalar Ö, Aydın A, 2004: The branches of the arteria celiaca in Badger. Indian Vet J, 183-187.

Yousefi MH, 2017: Ramification of celiac artery in the pine marten (Martes martes). Iran J Veterinary Sci Technol, 8(2), 60-65.

*Bu çalışmanın özeti 1st International Veterinary Anatomy Congress' te (Sandıklı / TÜRKIYE), 13-16.09.2017 tarihlerinde poster olarak sunuldu.

${ }^{* *}$ Sorumlu Yazar: Gülseren Kırbaş Doğan

Kafkas Üniversitesi, Veteriner Fakültesi, Anatomi Anabilim Dalı, Kars, Türkiye.

e-mail: glsrn36@gmail.com 